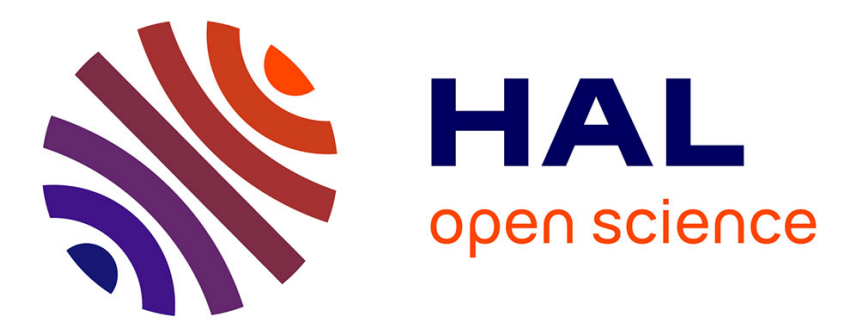

\title{
Non-Dominated Sorting Binary Differential Evolution for the Multi-Objective Optimization of Cascading Failures Protection in Complex Networks
}

Yan-Fu Li, Giovanni Sansavini, Enrico Zio

\section{- To cite this version:}

Yan-Fu Li, Giovanni Sansavini, Enrico Zio. Non-Dominated Sorting Binary Differential Evolution for the Multi-Objective Optimization of Cascading Failures Protection in Complex Networks. Reliability Engineering and System Safety, 2013, 111, pp.195-205. 10.1016/j.ress.2012.11.002 . hal-00750045

\section{HAL Id: hal-00750045}

https://hal-centralesupelec.archives-ouvertes.fr/hal-00750045

Submitted on 8 Nov 2012

HAL is a multi-disciplinary open access archive for the deposit and dissemination of scientific research documents, whether they are published or not. The documents may come from teaching and research institutions in France or abroad, or from public or private research centers.
L'archive ouverte pluridisciplinaire HAL, est destinée au dépôt et à la diffusion de documents scientifiques de niveau recherche, publiés ou non, émanant des établissements d'enseignement et de recherche français ou étrangers, des laboratoires publics ou privés. 


\title{
Non-Dominated Sorting Binary Differential Evolution for the Multi-Objective Optimization of Cascading Failures Protection in Complex Networks
}

\author{
Y.F. Li ${ }^{a^{*}}$, G. Sansavini ${ }^{\mathrm{b}}$, E. Zio ${ }^{\mathrm{a}, \mathrm{b}}$ \\ ${ }^{a}$ Chair on Systems Science and the Energetic Challenge, European Foundation for New Energy-Electricite' \\ de France, at Ecole Centrale Paris-Supelec, Paris, France \\ ${ }^{\mathrm{b}}$ Dipartimento di Energia, Politecnico di Milano, Milano, Italy
}

\begin{abstract}
A number of research works have been devoted to the optimization of protection strategies (e.g. transmission line switch off) of critical infrastructures (e.g. power grids, telecommunication networks, computer networks, etc) to avoid cascading failures. This work aims at improving a previous optimization approach proposed by some of the authors [1], based on the modified binary differential evolution (MBDE) algorithm. The improvements are three-fold: 1) in the optimization problem formulation, we introduce a third objective function to minimize the impacts of the switching off operations onto the existing network topology; 2) in the optimization problem formulation, we use the final results of cascades, rather than only a short horizon of one step cascading, to evaluate the effects of the switching off strategies; 3 ) in the optimization algorithm, the fast non-dominated sorting mechanisms are incorporated into the MBDE algorithm: a new algorithm, namely non-dominated sorting binary differential evolution algorithm (NSBDE) is then proposed. The numerical application to the topological structure of the $380 \mathrm{kV}$ Italian power transmission network proves the benefits of the improvements.
\end{abstract}

Keywords: complex networks, cascading failures, line switching, multi-objective optimization, differential evolution, fast non-dominated sorting

\section{LIST OF ABBREVIATIONS}

CI Critical infrastructure

DE Differential evolution

HV Hyper volume

MBDE Modified binary differential evolution

MOEA Multi-objective evolutionary algorithm

NSBDE Non-dominated sorting binary differential evolution

NP Network protection

NSGA-II Non-dominated sorting genetic algorithm-II

OPA Oak Ridge-PSERC-Alaska

PSA Power system analyzer

TAM Texas A\&M 


\section{INTRODUCTION}

In today's deregulated markets, critical infrastructures (CIs) (e.g. electric power grids, telecommunication networks, transportation networks, etc) are often run under stressful conditions which render their components more sensitive and vulnerable to natural and/or man-made disturbances $[2,3]$. In such systems, failure of one component may lead to a cascade of failures of other components and this can result in serious economical and social damages, as shown, for example, by recent large-scale blackouts of power grids $[4,5]$.

To confront this challenge, a number of analytical/simulation models have been built to anticipate the impact of cascading failures in complex networks. These models can be classified into three groups: 1) probabilistic models (e.g. CASCADE [6] and branching processes [7]); 2) complex network models (e.g. dynamic flow model [8], degree distribution model [9-12], and shortest path routing model [13, 14]); 3) physical simulation models (e.g. Oak Ridge-PSERC-Alaska (OPA) model [15], and Power System Analyzer (PSA) model [16], and Texas A\&M (TAM) model [17]). The first group of probabilistic models does not rely on the topological characteristics of the network (e.g. degree, centrality, etc). The second group resorts to graph theory to represent the topological properties of the components (nodes or links), which are used to describe the loads, and those of the whole network, which are used to define the failure redistribution mechanisms. The third group sets itself to account for both the network topology and the physical character of the actual systems (e.g. in the case of electrical power network, the power injection and flow according to circuit laws [18]).

The second group of models has been proposed for the vulnerability analysis of CIs [1, 3, 19-23] to: (1) identify preliminary vulnerabilities of CIs by topology-driven and dynamic analyses; (2) direct and focus further detailed analyses on the most critical areas of the CIs. Complex network models can also be practical in devising and verifying network protection (NP) techniques aimed at hampering the propagation of cascading failures. Such NP techniques are broadly divided in: (1) network interdiction [24-30], which enhances the network protection by designing/enhancing components and/or allocating redundancies to avoid failures, and (2) line switching [31-36], which hinders the failure propagation by cutting off the possible 'directions' along which the failures can spread within the network. Within the complex networks frame of work, line switching amounts to disconnecting edges (e.g. removing lines in a power system, closing a road in a transportation system or a pipe in a fluid distribution network, or disconnecting a router in an information system) in order to avoid or alleviate component overloads that may arise from the occurrence of failures in the infrastructure, which hence may initiate a cascading failure process. Such a strategy may be employed by an infrastructure either as a manual strategy or as an implemented automatic control strategy, in order to re-route flows so that heavily congested components are relieved. Line switching has been shown capable of dealing with cascading failures by proactive and economical actions that can be implemented immediately after the cascading initiation [1,32]. The success of this technique relies on the search for the optimal set of lines to be switched-off, with the objective of minimizing the extent of cascading failures $[1,13,37]$.

In a previous work [1], some of the authors have considered a two-objective optimization framework to minimize the connectivity losses in the system at both global and local levels. In the present work, a third 
objective is included to minimize the number of lines being switched off in order to reduce the impact of the switch-off operations onto the topology of the existing network and the associated costs. Indeed, after the switch-off is performed, the resulting connectivity reduction may often weaken the network leaving it even more vulnerable than before to further attacks or failures.

To solve the optimization problem for line switching, which is large-scale, nonlinear and combinatorial in nature, we propose a multi-objective evolutionary algorithm (MOEA), namely non-dominated sorting binary differential evolution (NSBDE) algorithm, whose novelty lies in incorporating the fast non-dominated sorting mechanisms [38] into the modified binary differential evolution algorithm (MBDE) [39]. DE is a population-based stochastic optimization technique [40] with successful applications in various scientific and engineering fields [41-43]. It is often reported that DE has superior performances than other evolutionary algorithms (e.g. genetic algorithms, particle swarm optimization) [44]. MBDE is a novel binary version of binary differential evolution (DE) [39] specialized on combinatorial problems.

The rest of this paper is organized as follows: Section 2 presents the cascading failure simulation model, the measure of cascading failure consequences, and the protection strategy, i.e. transmission line switching; Section 3 presents the general formulation of multi-objective optimization problems; Section 4 describes the detailed procedures of the proposed NSBDE algorithm; Section 5 presents the experiment results of NSBDE including the analyses and comparisons with the previous work [1]; Section 6 presents the discussions on the complex network modeling of cascading failures; Section 7 concludes this study and points out future research directions.

\section{CASCADING FAILURE MODEL AND PROTECTION STRATEGIES}

\subsection{Cascading failure model}

In general, the simulation of cascading failures in large-scale networks is computationally expensive. In order to focus on analyzing the failure propagation process and devising the prevention and/or mitigation measures, a complex network model has been developed by some of the authors [1]. The graph representation is used to abstract the electrical properties of the network, while retaining its structural properties. The representation is given in the form of an undirected graph $\boldsymbol{G}=(V, E)$ consisting of a set of $|V|$ nodes (substations or buses) and $|E|$ links (transmission lines). The elements in the set $V$ are divided into two subsets: $V_{G}$ generation nodes, i.e. sources of power, and $V_{D}$ distribution nodes, i.e. loads or substations. The network structure is described by an adjacency matrix $\left\{e_{i j}\right\}$ of the size $|V| \times|V|$ : if there is a link between node $i$ and node $j$, the entry $e_{i j}=1$, otherwise $e_{i j}=0$. The power is assumed to flow along the generator-distributor shortest paths $[13,19,37,45,46]$.

The load (or stress) on a network component, e.g. a node or transmission line, is modeled as dependent on the number of shortest paths transiting through it, when the power flow is sent from the available generation nodes to the distribution nodes. More precisely, the load, $L_{i}$ of node $i$ is measured by the node betweenness $[47,48]$, calculated as the fraction of the generator-distributor shortest paths passing through that node: 


$$
L_{i}=\frac{1}{(N-1)(N-2)} \sum_{i \in V_{G}, k \in V_{D}, j \in V, i \neq j \neq k} \frac{n_{i k}(j)}{n_{i k}}
$$

where $N=|V|$ is the total number of nodes in the graph $\boldsymbol{G}, n_{i k}$ is the number of shortest paths between the generator-distributor nodes, and $n_{i k}(j)$ is the number of generator-distributor shortest paths passing through the node $j$. Likewise, the load, $L_{i j}$ of a link $i j$, is measured by the edge betweenness, calculated as the fraction of the generator-distributor shortest paths through that link [49].

Each component in the network has a definite capacity, i.e. a maximum load it can sustain. The capacity of node $i$ (or link $i j$ ) is assumed to be proportional to its nominal load $L_{i}$ (or $L_{i j}$ ) at which it is designed to operate, $C_{i}=(1+\alpha) L_{i}$ (or $\left.C_{i j}=(1+\alpha) L_{i j}\right)$, where $\alpha \geq 0$ is the tolerance parameter assumed the same for all elements of the entire network. Despite the simplicity of the concept of $\alpha$, it can be regarded as an operating margin allowing safe operations of the components under possible load increments. When $\alpha=0$, the system is working at its limit capacity; any further load added to a component would result in its failure and, possibly, in cascading failures affecting a large portion of the network.

In the case that a component's load $L_{i}$ (or $L_{i j}$ ) exceeds its capacity $C_{i}$ (or $C_{i j}$ ), it is considered as failed, and thus, removed from the network. This leads to a redistribution of the shortest paths in the network and, consequently, to a change in the loads of some working components. If the loads on some components exceed their capacities upon the redistribution of the shortest paths, the components fail and, consequently, a new redistribution follows. The process continues until there are no further failures or all the components are failed $[13,14]$.

\subsection{Measuring cascading failure consequences}

To measure the effects of cascading failures, the connectivity loss $C_{L}$ is used [13, 37]. It quantifies the decrease of the ability of distribution substations to receive power from generators:

$$
C_{L}=1-\frac{1}{N_{D}} \sum_{i=1}^{N_{D}} \frac{N_{G}^{i}}{N_{G}}
$$

where $N_{G}=\left|V_{G}\right|$ and $N_{D}=\left|V_{D}\right|$ represent the numbers of generators and substations in the initial state of the network respectively, and $N_{G}^{i}$ represents the number of generators able to supply power to distribution node $i$ after the cascade of failures takes place. $C_{L}$ is an indicator that measures the effects of cascading failures at the global system level. On the other hand, it fails to identify the critical locations to which power supply has to be guaranteed under any contingency. Therefore, a second, local indicator $C_{L_{A}}$ is proposed to measure the effects of cascading failures on an identified critical region A. It quantifies the decrease of the ability of the distribution substations within the area A to receive power supply from any generator in the whole network: 


$$
C_{L_{A}}=1-\frac{1}{N_{D_{A}}} \sum_{i=1}^{N_{D_{A}}} \frac{N_{G}^{i}}{N_{G}}
$$

where $N_{D_{A}}$ represents the number of distribution nodes within the area A at the initial state of the network, and $N_{G}^{i}$ represents the number of generation units able to supply power flow to distribution node $i$ in the area A, after the cascade of failures takes place.

The cascade size $S$ can also be used to quantify the effects of cascading failures. It is defined as the number of nodes that fail and are disconnected from the network at the end of the cascade propagation [1]. Due to the strong correlation between them (i.e. an increase of $S$ produces an increase of $C_{L}$ ) and importance of $C_{L}$ to the power engineers, we use $C_{L}$ to formulate the first two objectives of the optimization problem.

\subsection{Cascading failure protection strategy and definitions of the objective functions}

In this work, the NP is modeled as an operator intervention targeting at minimizing the effects of the cascade failure propagation at both system and local levels by switching off a set of transmission lines immediately after a cascading failure is triggered. Due to the rapid unfolding of a cascading failure, it is assumed that the protection intervention takes place only once after the cascade is triggered: no further actions to correct the effects of this only protective action are taken [1]. Then, the problem arises of what is the best set of lines whose disconnection hinders the cascade propagation at the maximum while maintaining the minimum impact on the topology of the existing network. This issue is crucial because the intentional disconnection of lines may worsen the effects of the cascade in the same way as failure propagation does.

As previously explained, the two indicators, $C_{L}$ and $C_{L_{A}}$, can be used to quantify the effects of cascading failures. The global protection optimization seeks interventions that minimize the objective function $f_{1}(\boldsymbol{x})=C_{L}(\boldsymbol{x})$, i.e. the connectivity loss of the line switch-off set $\boldsymbol{x}=\left\{x_{j}\left|x_{j} \in\{0,1\}, j=1, \ldots, K, K=\right| E \mid\right\}$, where $x_{j}=1$ if link $j$ is switched-off, or 0 otherwise. Secondly, the local optimization searches for the optimal intervention that minimizes the connectivity loss $C_{L_{A}}$ of a specific area $\mathrm{A}, f_{2}(\boldsymbol{x})=C_{L_{A}}(\boldsymbol{x})$. Thirdly, to reduce the impact of the mitigation operations, the number of lines being switched-off is also minimized, $f_{3}(x)=\sum_{j=1}^{K} x_{j}$.

In order to fully take advantage of all the perspectives on network protection mentioned above, we look into a "hybrid" protection strategy that finds the set of lines to be switched-off, $\boldsymbol{x}$, that minimize the connectivity loss of a pre-identified area $\mathrm{A}, C_{L_{A}}(\boldsymbol{x})$, the connectivity loss of the whole network, $C_{L}(\boldsymbol{x})$, and the number of lines being switched-off, $\sum_{j=1}^{K} x_{j}$. This originates a multi-objective optimization problem, which is here tackled by a MOEA as is detailed in Section 4. The algorithm for simulating the combined effects of cascading failures and protection strategies proceeds in successive stages as follows; it will be used for the fitness evaluation in the MOEA:

step 1. Receive all the parameters used to characterize the current topology (after the initial attack) of the network $\boldsymbol{G}$ and the protection strategy. The parameters include the set of functioning nodes $V$ and set of functioning links $E$, the load of each node $L_{i}$ and the load of each link $L_{i j}$, the maximum capacity 
of each node $C_{i}$ and the maximum capacity of each link $C_{i j}$, and the vector $\boldsymbol{x}$ indicating the lines to be switched-off. Set the stage counter $m=0$. It is noted that by setting $\boldsymbol{x}$ to be a zero vector, this algorithm is able to simulate the cascading failures without any protection operations.

step 2. Remove a set of links indicated by $\boldsymbol{x}$ and apply Floyd's shortest paths algorithm [50] to compute the load of each node (see eq. (1)) and link.

step 3. Test each component for failure: for $i=1, \ldots, N$, if node $i$ is working and $L_{i}>C_{i}$ then node $i$ is removed; if link $i j$ is working and $L_{i j}>C_{i j}$ then link $i j$ is removed. Increase the stage counter $m$ by 1 .

step 4. Evaluate the load of each component using Floyd's shortest paths algorithm. Then each component that is operating is tested for failure: for $i=1, \ldots, N$, if node $i$ is working and $L_{j}>C_{j}$ then node $i$ fails; if link $i j$ is working and $L_{i j}>C_{i j}$ then link $i j$ fails.

step 5. If any working node fails, then increase $m$ by 1 and return to step 4 . Otherwise, terminate the algorithm.

To simulate the initiating event, i.e. the failure of the most critical component in terms of load, some components need to be removed from the network prior to the execution of the algorithm presented above.

The adopted protection strategy differs from the one presented in [1]. In particular, the former identifies solutions that are optimum with respect to $C_{L}$ and $C_{L_{A}}$ values at the end of the cascade propagation, while the latter identifies solutions that are optimum with respect to $C_{L}$ and $C_{L_{A}}$ values at the step immediately following protection, i.e. $t=1$, and the propagation of the cascade following the step $t=1$ is not accounted for in the optimization algorithm. Furthermore, the latter does not include the number of lines to switch off as an objective of the optimization.

\section{GENERAL FORMULATION OF MULTI-OBJECTIVE OPTIMIZATION}

Real world applications involve the simultaneous optimization of several objective functions, which are often competing or/and conflicting with one another, and subject to a number of equality and inequality constraints. In general, these multi-objective problems can be formulated as follows (in terms of minimization):

$$
\begin{array}{ll}
\text { Minimize } & f_{o}(\boldsymbol{x}), o=1, \ldots, O \\
\text { Subject to } & \begin{cases}g_{l}(\boldsymbol{x})=0, & l=1, \ldots, L \\
h_{m}(\boldsymbol{x}) \leq 0, & m=1, \ldots, M\end{cases}
\end{array}
$$

where $f_{o}$ is the $o$-th objective function, $\boldsymbol{x}$ is a decision vector that represents a solution, $O$ is the number of objectives, $g_{l}$ is the $l$-th of the $L$ equality constraints and $h_{m}$ is the $m$-th of the $M$ inequality constraints. The objective functions $f_{o}(\boldsymbol{x})$ must be evaluated in correspondence of each decision variable vector $\boldsymbol{x}$ in the 
search space. The final goal is to identify a set of optimal decision variable vectors $\boldsymbol{x}_{p}^{*}, p=1,2, \ldots, P$, instead of a single optimal solution. In this set of optimal solutions, no one can be regarded to be better than any other with respect to all the objective functions. The comparison of solutions may be achieved in terms of the concepts of Pareto optimality and dominance [51]: in case of a minimization problem, solution $\boldsymbol{x}_{a}$ is regarded to dominate solution $\boldsymbol{x}_{b}\left(\boldsymbol{x}_{a}>\boldsymbol{x}_{b}\right)$ if both following conditions are satisfied:

$$
\begin{aligned}
& \forall i \in\{1,2, \ldots, O\}, f_{i}\left(\boldsymbol{x}_{a}\right) \leq f_{i}\left(\boldsymbol{x}_{b}\right) \\
& \exists j \in\{1,2, \ldots, O\}, f_{j}\left(\boldsymbol{x}_{a}\right)<f_{j}\left(\boldsymbol{x}_{b}\right)
\end{aligned}
$$

If any of the above two conditions is violated, the solution $\boldsymbol{x}_{a}$ does not dominate the solution $\boldsymbol{x}_{b}$, and $\boldsymbol{x}_{b}$ is said to be non-dominated by $\boldsymbol{x}_{a}$. The solutions that are non-dominated within the entire search space are denoted as Pareto-optimal and constitute the Pareto-optimal set, and the corresponding values of the objective functions form the so called Pareto-optimal front in the objective functions space. The goal of a multi-objective optimization algorithm is to guide the search for solutions in the Pareto-optimal set, while maintaining diversity so as to cover well the Pareto-optimal front and thus allow flexibility in the final decision on the solutions to be actually implemented.

\section{NON-DOMINATED SORTING BINARY DIFFERENTIAL EVOLUTION ALGORITHM}

In this Section, we present the operation procedures of the NSBDE algorithm. DE has been originally proposed as a population-based global optimization algorithm for real-valued optimization problems [40]. The standard DE algorithm is simple and efficient and has been successfully applied in various scientific and engineering fields [41-43] often with superior performance than alternative optimization algorithms, e.g. particle swarm optimization and genetic algorithms [44]. Modified binary differential evolution (MBDE) is a binary version of DE developed to tackle single-objective binary-coded optimization problems [39]. In order to solve the combinatorial multi-objective problem of interest, we introduce into MBDE the fast nondominated sorting, ranking, and elitism techniques utilized in non-dominated sorting genetic algorithm-II (NSGA-II) [38]. The NSBDE algorithm proceeds with the following steps:

\section{Step 1. Initialization of parameters}

Define the values of: the population size $N P$, the crossover rate $C R$, the scaling factor $F$, and the maximum number of generations $N_{\max }$.

\section{Step 2. Generation of initial population and evaluation}

Set the generation number $t$ equal to 1 . Initialize the population $\boldsymbol{X}_{t}=\left\{\boldsymbol{x}_{1}^{t}, \ldots, \boldsymbol{x}_{M}^{t}\right\}$ which contains $M$ realvalued parameter vectors of length $K$. Each vector is also called a chromosome and forms a candidate solution to the optimization problem. Each element of each vector $x_{i j}^{t}=\left\{x_{i j}^{t} \mid x_{i j}^{t} \in\{0,1\}\right\}, i=1,2, \ldots, M$, 
$j=1, \ldots, K$ takes a value $\left(b_{i j}\right)$ from the set $\{0,1\}$ with probability equal to 0.5 : the element takes value ' 1 ' if the corresponding line is to be switched-off, 0 otherwise. Evaluate each of the $M$ chromosomes in the initial population $\boldsymbol{X}_{t}$ by performing the cascading failure protection simulation algorithm presented in Section 2.3. Return the values of the two indicators of the connectivity loss, $C_{L}$ and $C_{L_{A}}$. The number of switched-off lines can be directly obtained by $\sum_{j=1}^{K} x_{i j}^{1}$.

\section{Step 3. Generation of intermediate population}

Apply the binary tournament selection operator [38] to the population $\boldsymbol{X}_{t}$ to generate a trial population $\boldsymbol{X}_{t}^{\prime}=\left\{\boldsymbol{x}_{1}^{\prime t}, \ldots, \boldsymbol{x}_{M}^{\prime t}\right\}$, which undergoes the evolution operations of mutation and crossover to become an intermediate population $\boldsymbol{V}_{t}=\left\{\boldsymbol{v}_{1}^{t}, \ldots, \boldsymbol{v}_{M}^{t}\right\}$.

\section{Step 3.1 Mutation}

Apply the mutation operator (7) onto each binary chromosome of $\boldsymbol{X}_{t}^{\prime}[39]$ :

$$
P\left(x_{i j}^{\prime t}\right)=1 /\left(1+\exp \left(\frac{2 b\left[x_{r_{1}, j}^{\prime t}+F\left(x_{r_{2}, j}^{\prime t}-x_{r_{3}, j}^{\prime t}\right)-0.5\right]}{1+2 F}\right)\right) \text { where } j=\{1, \ldots, K\}
$$

where $b$ is a positive real constant, often set to values around $6, F$ is the scaling factor, $x_{r_{1}, j}^{\prime t}, x_{r_{2}, j}^{\prime t}$, and $x_{r_{3}, j}^{\prime t}$ are the elements at the $j$-th position of the three randomly chosen chromosomes $\boldsymbol{x}_{r_{1}}^{\prime t}, \boldsymbol{x}_{r_{2}}^{\prime t}$, and $\boldsymbol{x}_{r_{3}}^{\prime t}$, with indexes $r_{1} \neq r_{2} \neq r_{3} \neq i$. After applying (7) onto the current chromosome, the noisy vector is generated as,

$$
u_{i j}^{t}=\left\{\begin{array}{rr}
1, & \text { if rand } \leq P\left(x_{i j}^{\prime t}\right) \\
0, & \text { otherwise }
\end{array}\right.
$$

where rand is a uniformly distributed random number within the interval $[0,1]$.

\section{Step 3.2 Crossover}

Apply the crossover operator (9) to mix the noisy and target vectors to create an intermediate vector $\boldsymbol{v}_{i}$. The vector inherits different pieces from the noisy and target vectors, as regulated by the crossover rate $C R$. The commonly used binomial crossover is defined as:

$$
v_{i j}^{t}=\left\{\begin{array}{ll}
u_{i j}^{t}, & \text { if } U(0,1] \leq C R \text { or } j=\operatorname{irand}(M) \\
x_{i j}^{\prime t}, & \text { if } U(0,1]>C R \text { or } j \neq \operatorname{irand}(M)
\end{array} \quad \forall j \in\{1,2, \ldots, K\}\right.
$$

where $U(0,1]$ is a uniform random value $\in(0,1] \operatorname{irand}(M)$ is a uniform discrete random number in the set $\{1,2, \ldots, M\}, j$ is the index of the dimensionality and $K$ is the length of the chromosome. 


\section{Step 4. Evaluation}

Evaluate each of the $M$ chromosomes in the population $\boldsymbol{V}_{t}$ by performing the cascading failure protection simulation algorithm presented in Section 2.3. Return the values of the two indicators of the connectivity loss, $C_{L}$ and $C_{L_{A}}$. The number of switched-off lines can be directly obtained by $\sum_{j=1}^{K} v_{i j}^{t}$.

\section{Step 5. Union and sorting}

Combine the parent and intermediate populations to obtain a union population $\boldsymbol{R}_{t}=\boldsymbol{X}_{t} \cup \boldsymbol{V}_{t}$. Rank the chromosomes in the population $\boldsymbol{R}_{t}$ by running the fast non-dominated sorting algorithm [38] with respect to the objective values, and identify the ranked non-dominated fronts $F_{1}, F_{2}, \ldots, F_{k}$ where $F_{1}$ is the best front, $F_{2}$ is the second best front and $F_{k}$ is the least good front.

\section{Step 6. Selection}

Select the first $N P$ chromosomes from $\boldsymbol{R}_{t}$ to create a new parent population $\boldsymbol{X}_{t+1}$. The crowding distance measure is used in this step to compare the chromosomes with the same rank (a more 'crowded' chromosome has lower priority than a less 'crowded' one), where crowding refers to the density of solutions present in a neighborhood of a chromosome of specified radius [38]. Increase the generation number $t$ by 1 . Stop the algorithm if $t \geq N_{\max }$ otherwise go to Step 3 .

\section{CASE STUDY AND RESULTS}

\subsection{Case study description and cascading failures without interventions}

The proposed optimization method for network protection strategies is exemplified with reference to the topological network of the $380 \mathrm{kV}$ Italian power transmission network (Figure 1), focusing only on its structure with no further reference on the electrical properties. This network is a branch of a high voltage level transmission, which can be modeled as a graph of $N=127$ nodes $\left(N_{G}=30\right.$ generators and $N_{D}=97$ distributors) and $K=171$ edges. In Figure 1, the generators, i.e. hydro and thermal power plants, are represented by squares whereas the distribution substations are represented by circles. As shown in Section 2.1, the load of each component is computed by Eq. (1). In a previous analysis by some of the authors [1], it was found that the removal of link 107 (connecting the nodes 78 (Bargi Stazione) and 81 (Calenzano)) results in the largest damage in terms of $C_{L}=0.96$ and failure size (57 nodes fail at the end of cascading). Additionally, node 78 was found to be one of the most critical nodes in initiating the cascading failure propagation.

Since the region of Lombardy includes the largest number of distribution nodes in the power grid (densely-dotted area in the upper side of Figure 1), i.e. 21 distributors, the local protection strategy focus on the minimization of the loss of connectivity of this specific area, $C_{L_{A}}$. 


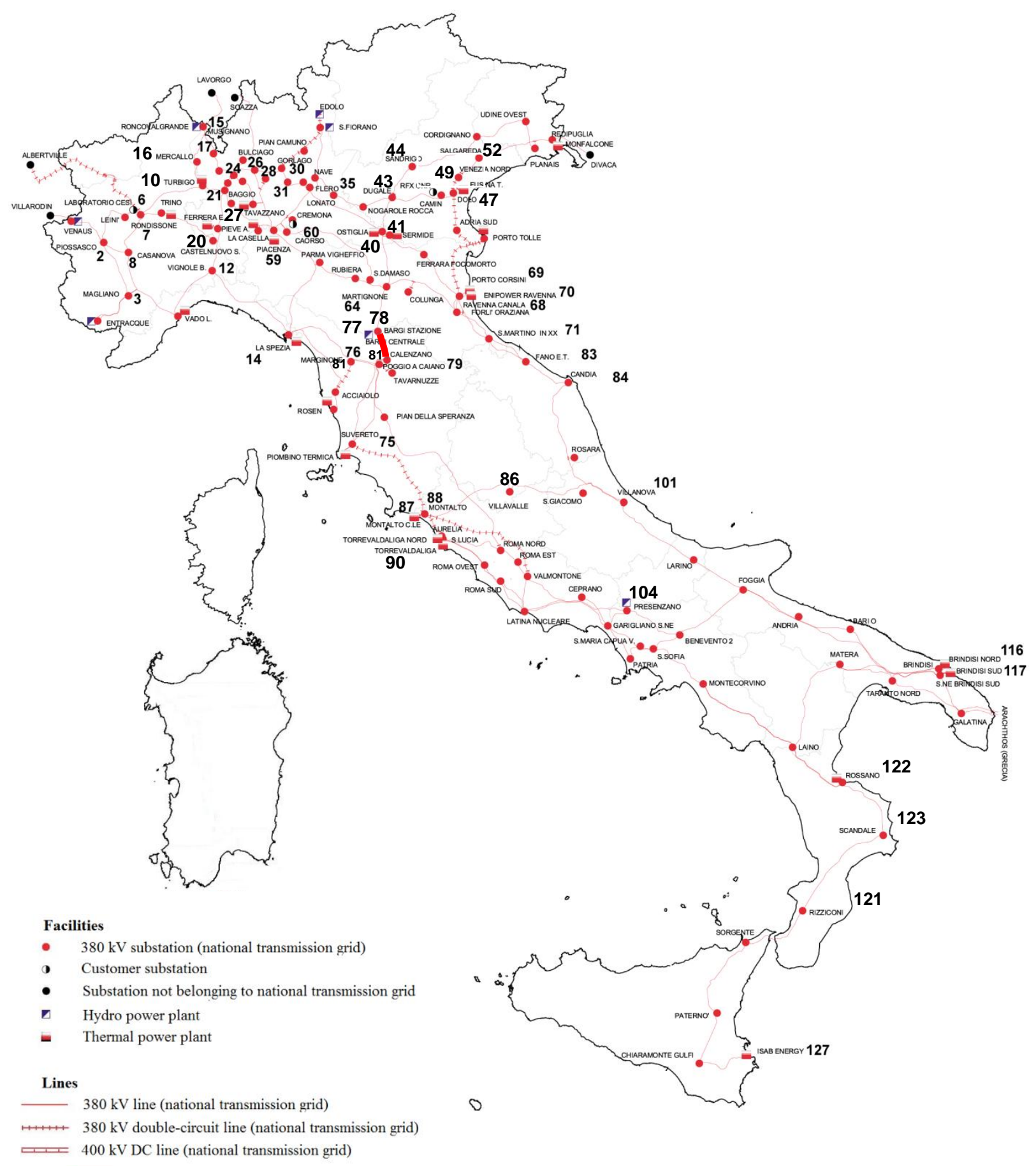

Figure 1. The $380 \mathrm{kV}$ Italian power transmission network [52]

Figure 2 illustrates the evolution of the cascade from the initial failure until the end of cascading, when no intervention is performed. The tolerance parameter of the cascading failure model $\alpha$ is set to 0.3 , in line with the previous work [1]. The elements in black color experience load changes beyond their capacity at the current cascading stage. It is shown that a large number of elements are overloaded (Figures 2.a and 2.b) and fail after the initial attack, and are removed from the network (Figures 2.b and 2.c). Conversely, the loads of some elements decrease after the initial attack. Nonetheless, in the following steps these elements exceed the maximum capacities and are removed from the network due to the further propagation of the cascade. The 
cascade stops after the stage $t=2$ (Figure 2.c). At the end of the cascade, the network is clustered into several islands (Figure 2.d).

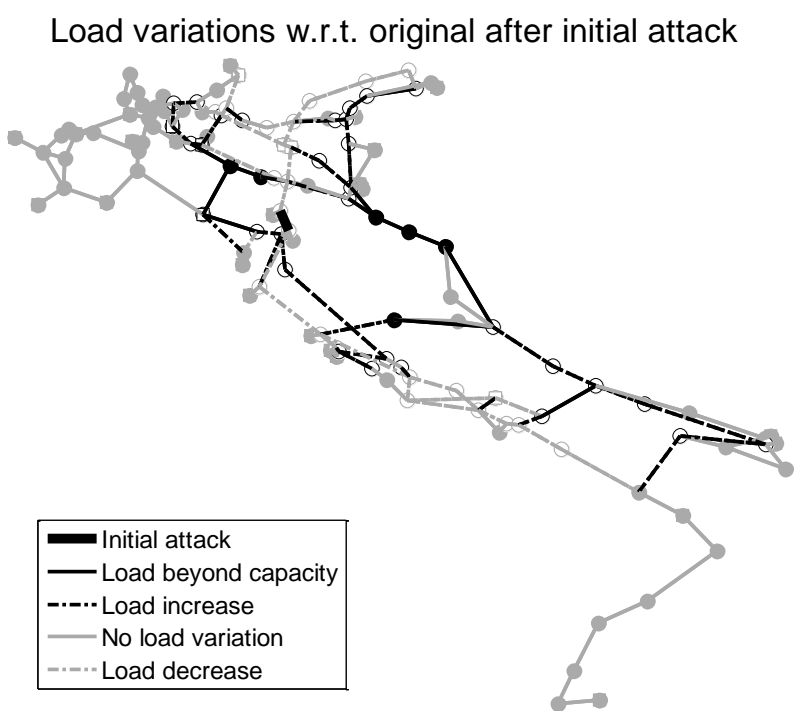

(a)

Load variations at step 2 w.r.t. previous step
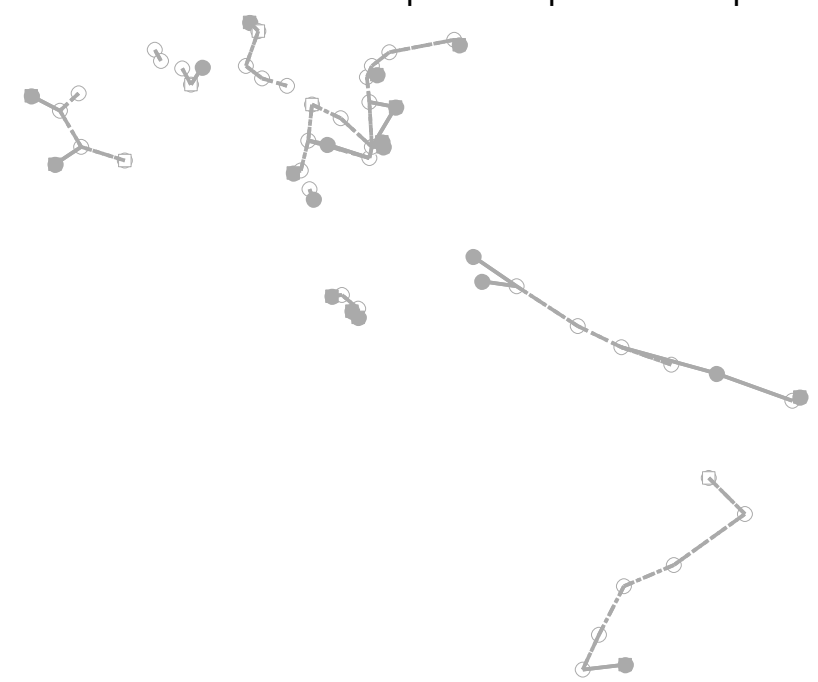

(c)

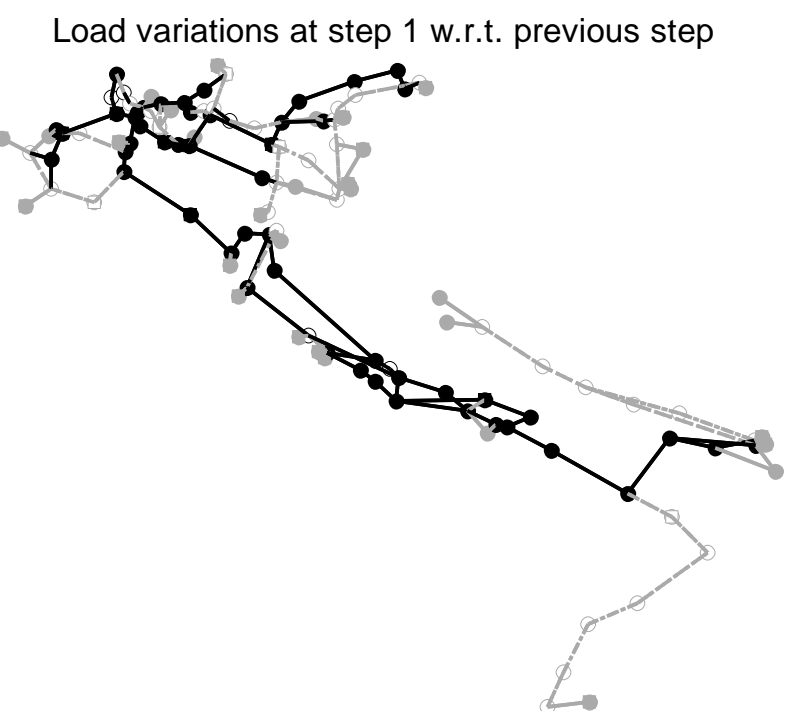

(b)

Load variations at step 3 w.r.t. previous step
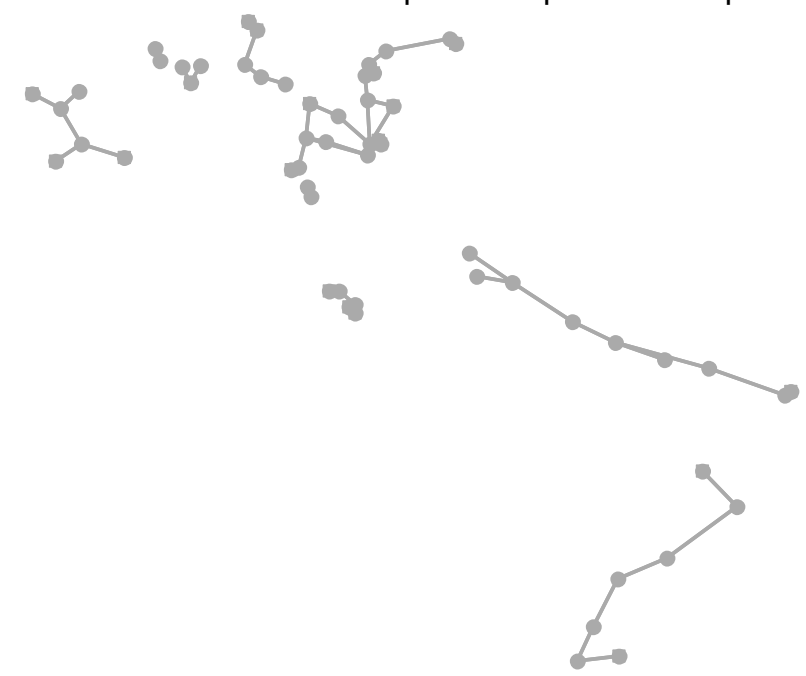

(d)

Figure 2. Illustration of the cascading failure propagation steps after the initial removal of line 107 and no intervention

\subsection{Results of the NSBDE algorithm}

According to the protection strategy presented in Section 2.3, it is assumed that the protection intervention (line switching) takes place only once when $t=0$ after the failure of the transmission line 107. The configurations of the NSBDE parameters are summarized in Table 1. The NSBDE parameters and the number of experiment runs (equal to 5) are set identical to those in [1]. 
Table 1. The parameters of the NSBDE algorithm

\begin{tabular}{|l|l|}
\hline \multicolumn{2}{|c|}{ NSBDE parameters } \\
\hline Population size $N P$ & 40 \\
\hline Dimensionality of solutions $K$ & 171 \\
\hline Crossover rate $C R$ & 0.8 \\
\hline Scaling factor $F$ & 0.2 \\
\hline Minimum fitness error & $10^{-4}$ \\
\hline Maximum number of generations & 1500 \\
\hline
\end{tabular}

The convergence plots of one experiment run of NSBDE algorithm are shown in Figure 3. The three panels in Figure 3 show the three extreme points of the best Pareto-front at each generation during the evolution (namely, the minimum global connectivity loss, the minimum local connectivity loss, and the minimum number of lines switched-off). It is observed that NSBDE is able to converge after about 600 generations.
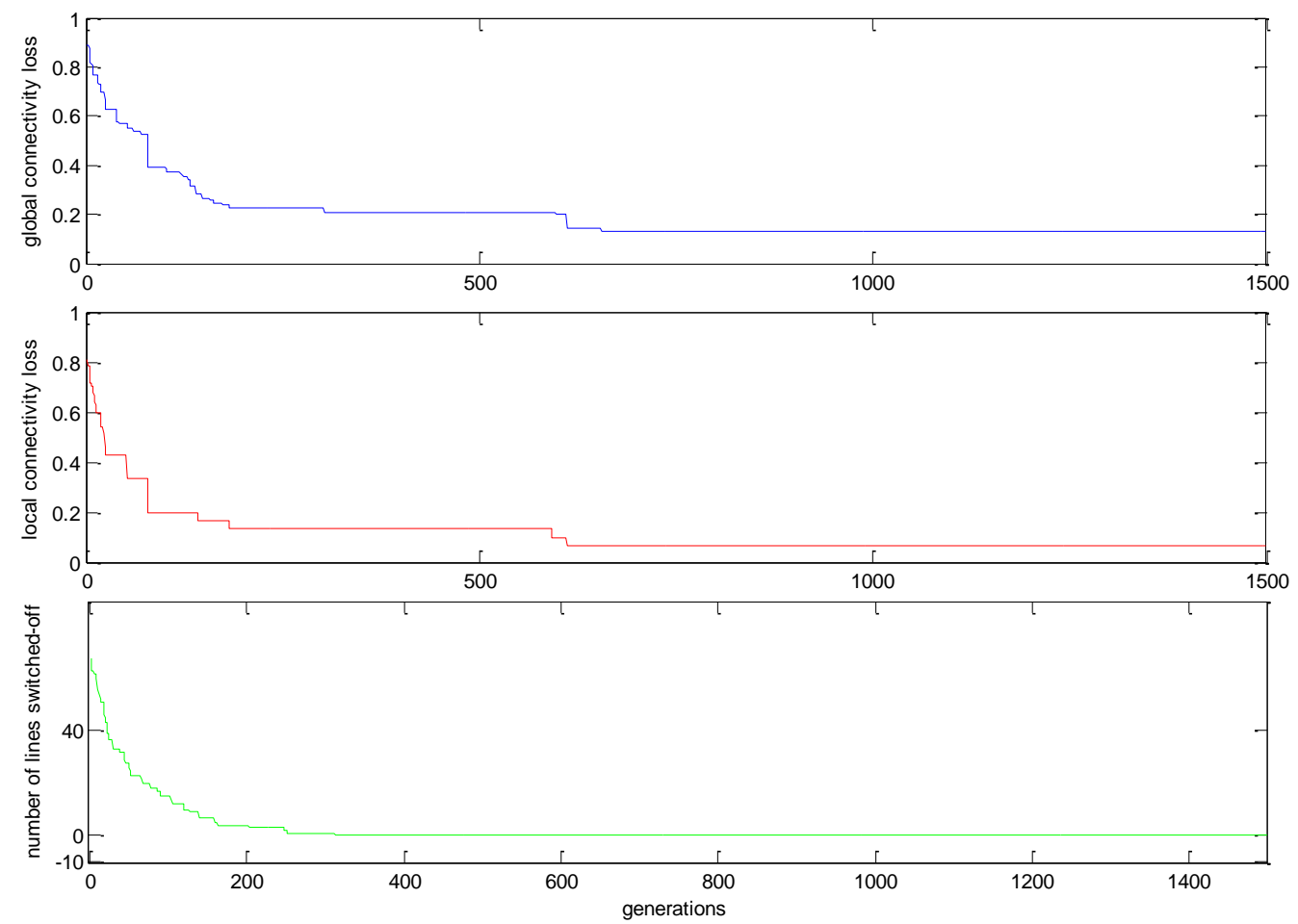

Figure 3. The convergence plots of NSBDE algorithm

The quality of the estimated Pareto front at each experiment run is assessed by the well-known hyper volume (HV) [53] measure. HV is defined as the volume or hyper volume between the estimated Pareto front and a predefined reference point. The exact computation of HV can be usually time-consuming; here, we resort to a MATLAB tool based on the Monte Carlo method [54]. The two parameters for HV calculation, the number of sampled random points and the reference point, are set to $10^{7}$ and $(1,1,4)$, respectively. The mean value of the five HVs is 0.2771 , the standard deviation is 0.0557 , the maximal HV is 0.3276 , and the minimal HV is 0.2175 . 
The final estimated Pareto front obtained by selecting the non-dominated solutions among those reached in the five different runs is illustrated in the 3-D space of Figure 4.a. The HV of this front is 0.3278, higher than any of the 5 individual fronts. The three 2-D projections of the solutions are shown in Figures 4.b to 4.d, respectively.

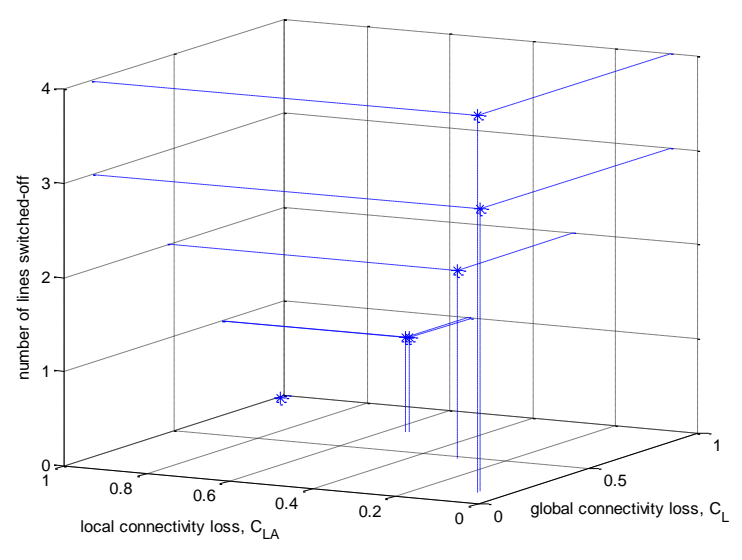

(a)

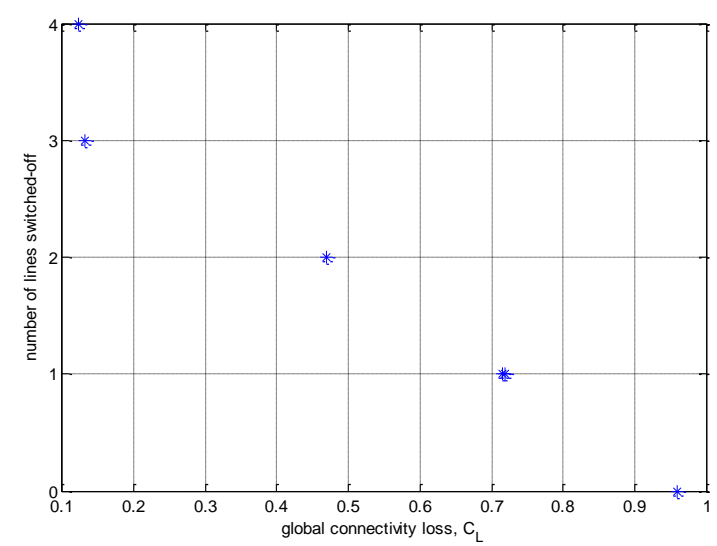

(c)

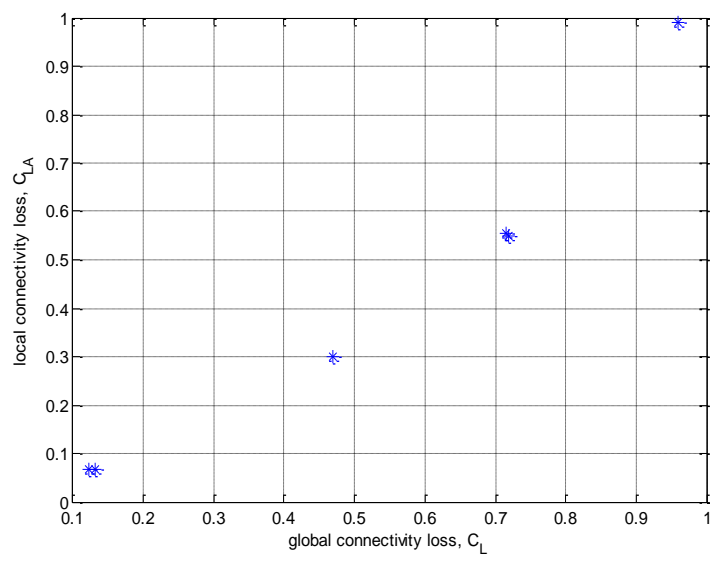

(b)

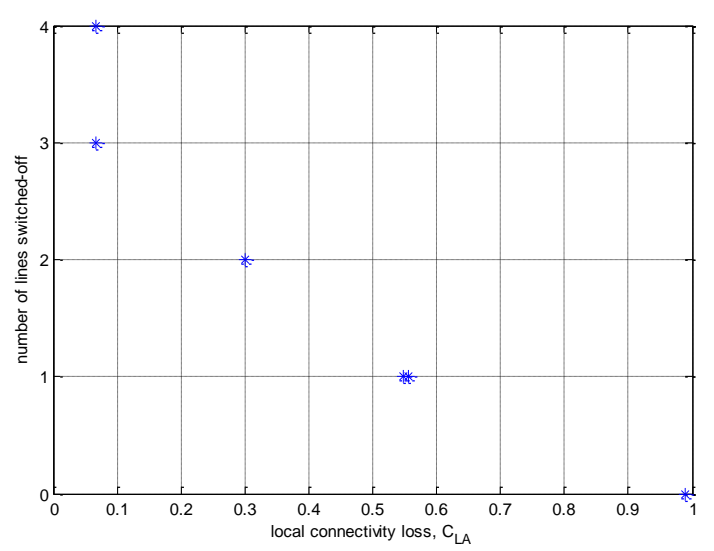

(d)

Figure 4. The Pareto front in 3-D space and its 2-D projections

The numerical results of these strategies in terms of the three objective functions, $C_{L}, C_{L_{A}}$ and $\sum_{j=1}^{K} x_{j}$ (i.e. the solutions in the estimated Pareto front) are presented in Table 2. They are non-dominated to each other in terms of the three objectives. However, strategy \#5 is of particular interest since it has significant smaller $C_{L}$ and $C_{L_{A}}$ values than the other solutions, except strategy \#6, and it requires switching off a set of only 3 transmission lines, less than in strategy \#6.

Table 2. The non-dominated solutions of 5 NSBDE experiment runs

\begin{tabular}{|c|c|c|c|}
\hline Strategy ID & $C_{L}$ & $C_{L_{A}}$ & $\sum_{j=1}^{K} x_{j}$ \\
\hline 1 & 0.959 & 0.990 & 0 \\
\hline 2 & 0.719 & 0.548 & 1 \\
\hline 3 & 0.715 & 0.556 & 1 \\
\hline
\end{tabular}




\begin{tabular}{|l|l|l|l|}
\hline 4 & 0.469 & 0.300 & 2 \\
\hline 5 & 0.132 & 0.067 & 3 \\
\hline 6 & 0.122 & 0.067 & 4 \\
\hline
\end{tabular}

\subsection{Analyses and interpretation of the results}

Strategy \#5 is selected for the illustration of cascades development considering the optimal operator interventions. Figure 5 contains the 'snap-shot' of the cascading failure process at each stage from the initial attack until its end. The state of the network following the initial attack presented in Figure 2.a and in Figure 5.a is the same. Several components are potentially overloaded and would be disconnected from the system if no protection was put in action (Figure 2.b). Unlike the scenario presented in Section 5.1, the protection is applied (Figure 5.b), and three links are switched off as part of the protection strategy at the stage $t=1$, i.e. links 43-44, 49-52, and 64-78. As the result of the protection strategy, the potential overloads are relieved and the cascading failure is successfully mitigated after these links are switched off (Figure 5.b). Figure 5.b shows that none of the elements exceeds the maximum capacity in this scenario. Therefore, there are no further disconnections in the network after the protection action is put in action (Figure 5.c), the cascade cannot propagate in the network and the failure process stops at $t=1$ (Figures 5.c and 5.d). Throughout the cascading failure process only 4 links are disconnected, including the initial removal, and no node is disconnected resulting in $S=0$, i.e. the cascade size is zero.

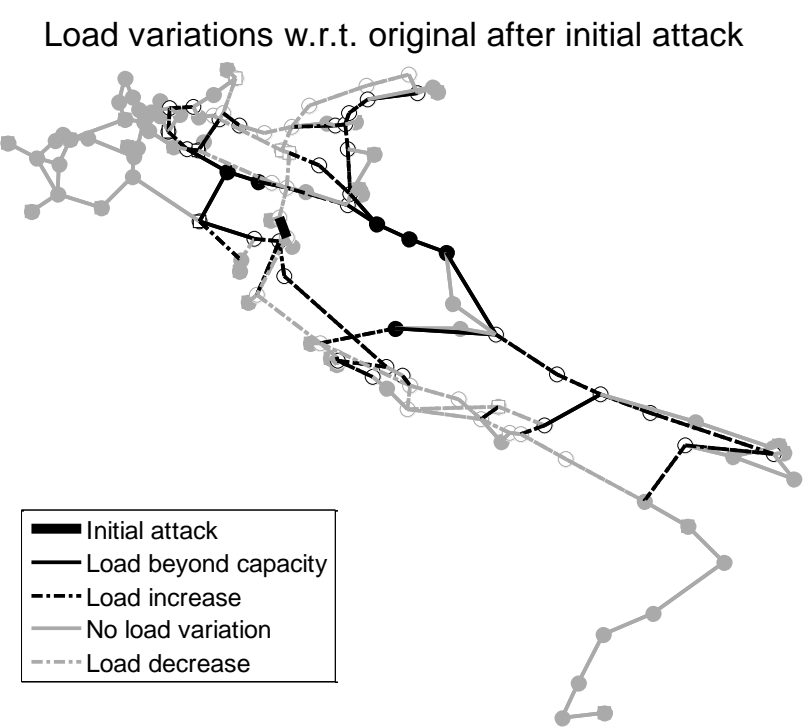

(a)

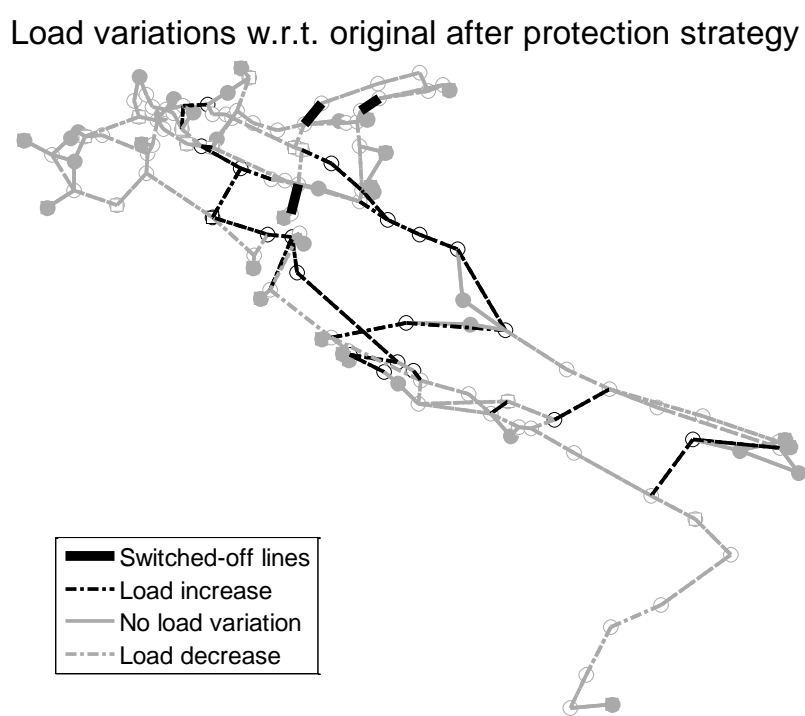

(b) 
Load variations at step 1 w.r.t. previous step

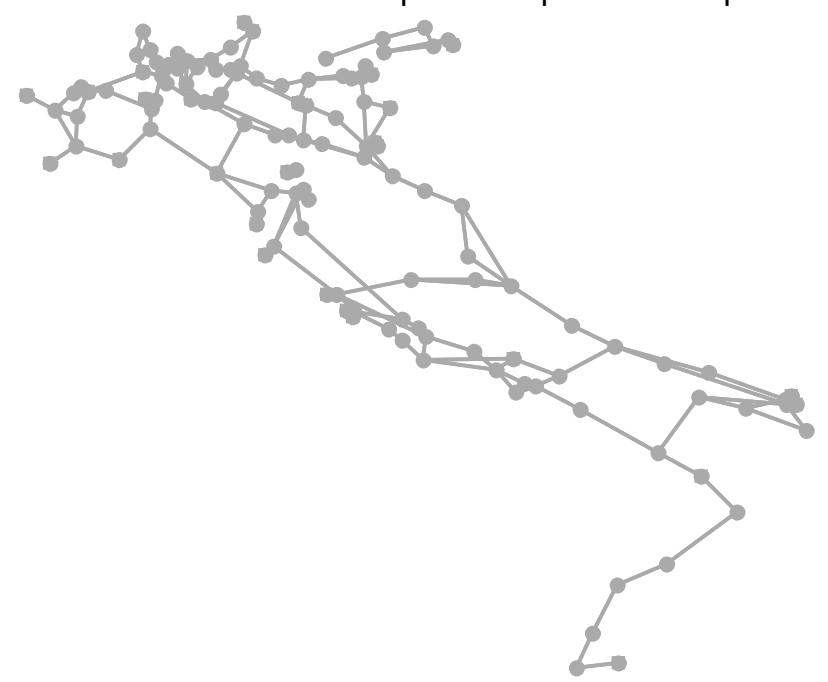

(c)

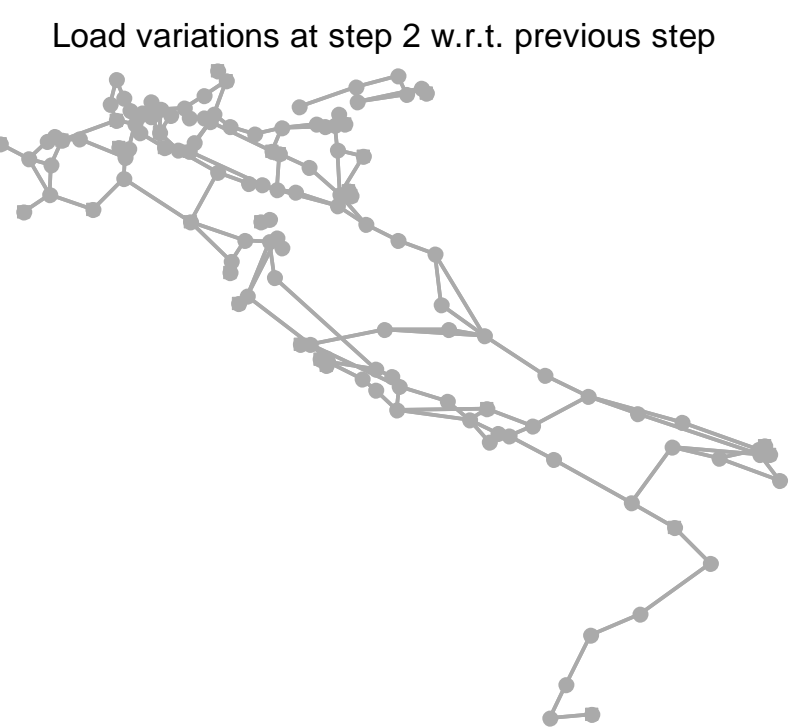

(d)

Figure 5. The illustration of cascading failure propagation after the failure of line 107 with optimal intervention strategy \#5

Figure 6 shows the effects of the operator optimal intervention by comparing the $C_{L}$ and $C_{L_{A}}$ values of the four optimal strategies in Table 2 at each stage of the cascade. We note that the NSBDE optimization identifies that no intervention can be a possible optimum protection strategy, indicated as strategy \#1, because it provides the global minimum of the number of lines to switch off. Among all strategies, strategy \#5 presents the best local connectivity loss and the second best global connectivity loss, at the end of the cascade failure after the protection strategy is applied at $t=1$. These results are consistent with the cascade evolution presented in Figure 5. Strategy \#1 (no protection) performs better than the other three protection strategies at $t=1$, but it allows the cascade to propagate and to affect the whole network with disruptive consequences. Conversely, strategies \#4, \#5 and \#6 are able to stop the failure process at $t=1$.
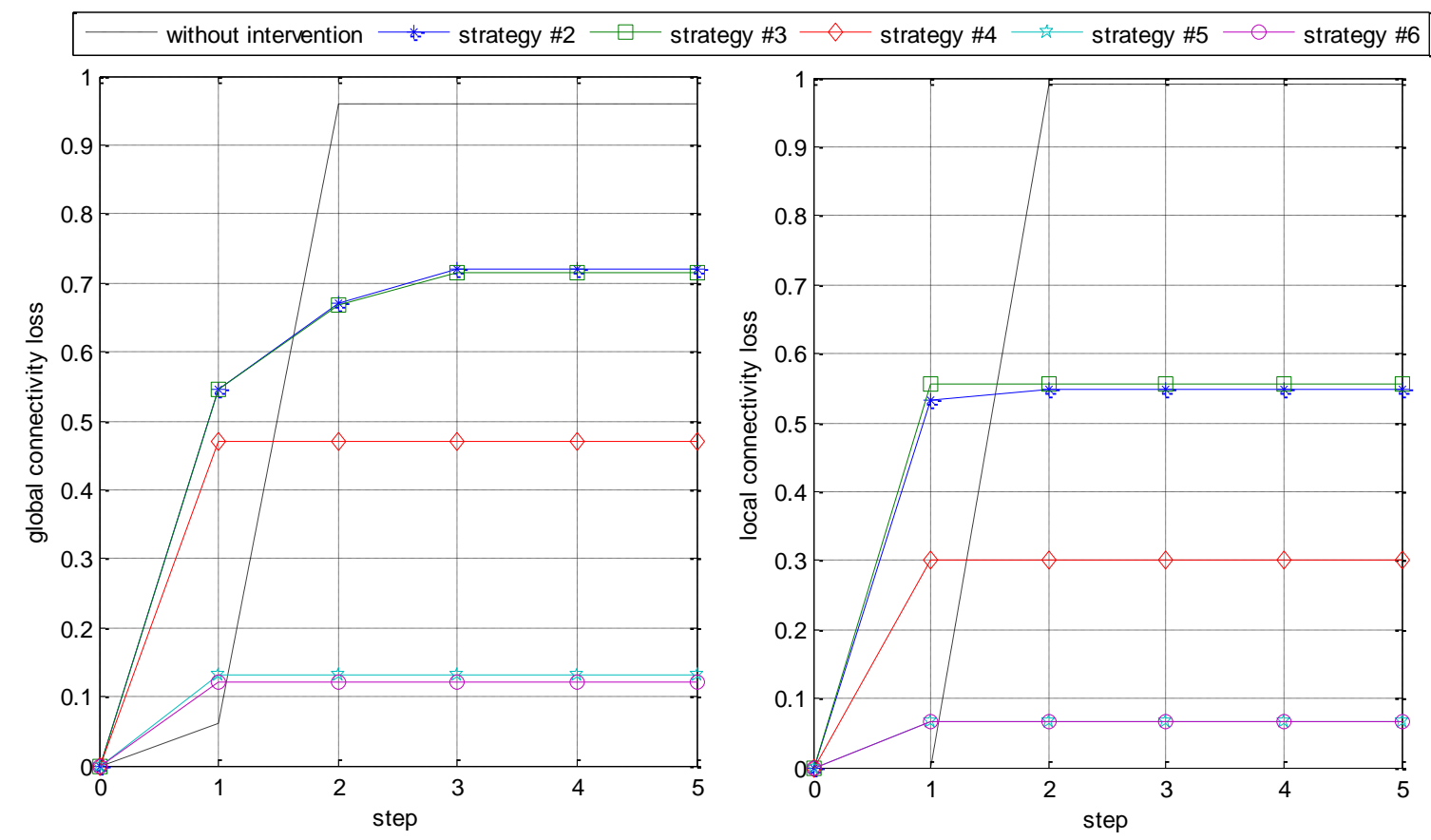
Figure 6. The cascade development in terms of global connectivity loss, $C_{L}$, and local connectivity loss, $C_{L_{A}}$, after the failure of line 107, with and without interventions

Looking at the previous results obtained in [1] we note that the effect of introducing the third objective of minimizing the number of lines to be switched off, of looking at the outcome of the whole cascades, of integrating non-dominated sorting mechanisms in the optimization search (Table 3), leads to strategies obtained by NSBDE which are in general superior in terms of the connectivity losses, the number of lines being switched off, and the cascade size. In particular, the NSBDE strategy \#3 and \#4 seem most promising and practical because they result in significantly smaller $C_{L}$ and $C_{L_{A}}$ values than those relative to the other strategies, and they require significantly lower numbers of switching off, i.e. $\sum_{j=1}^{K} x_{j}=3$ or 4 . Furthermore, the cascade size $S$ is equal to zero because no node is disconnected from the network at the end of the failure process.

Table 3. The results of the NSBDE and the results published in [1]

\begin{tabular}{|l|l|l|l|l|l|}
\hline \hline Method & Strategy ID & $C_{L}$ & $C_{L_{A}}$ & $\sum_{j=1}^{K} x_{j}$ & $S$ \\
\hline $\begin{array}{l}\text { No } \\
\text { intervention }\end{array}$ & 1 & 0.96 & 0.99 & 0 & 57 \\
\hline MBDE [1] & 1 & 0.76 & 0.81 & 31 & 10 \\
\hline & 2 & 0.77 & 0.89 & 26 & 13 \\
\hline & 3 & 0.75 & 0.80 & 30 & 9 \\
\hline NSBDE & 2 & 0.719 & 0.548 & 1 & 12 \\
\hline & 3 & 0.715 & 0.556 & 1 & 10 \\
\hline & 4 & 0.469 & 0.300 & 2 & 0 \\
\hline & 5 & 0.132 & 0.067 & 3 & 0 \\
\hline & 6 & 0.122 & 0.067 & 4 & 0 \\
\hline
\end{tabular}

\section{DISCUSSION}

We embrace the complex network perspective on the network protection (NP) problem. This perspective allows characterizing the topology of network systems and identifying critical parts by an analysis that is preliminary and complementary to more detailed approaches [55-58]. The feasibility of line switching is evaluated by a model of cascading failure process which relies on a topological representation of the network connection pattern [46]. The abstract model of flow and cascades is not based on the physics of the actual power flow but rather mimics the flow in the network based on the shortest paths that connect generation and load nodes.

Given the somewhat abstract level of the modeling, the results gained with respect to the vulnerable points (or lines) in the system (first findings) may not be clear-cut, and major hidden vulnerabilities may still be expected. Then, to achieve a higher degree of accuracy, system understanding has to be further developed and more detailed information about the system and its operating environment may be needed. The reassessment of simplifications made earlier may call for more sophisticated methods for their successive indepth analysis [59]. 
In order to draw firm conclusions about the significance of topological methods, e.g. in electricity infrastructure vulnerability characterization, current research focuses on systematically comparing their results with those obtained from power flow-based models $[52,57,60]$. The general feeling is that only a partial superposition of functional and structural criticalities should be expected. In this view, the abstract models of flow and cascading failures that are used in this study cannot be interpreted as an approximation of real, functional data.

The research presented, thus exemplifies the proposed methodology with reference to a power transmission network, focusing only on its structure and with no further reference on the physical and functional properties. As described in Section 2, the load of a node is assumed equal to the total number of shortest paths connecting every source node to every sink node passing through that component. This assumption may not be functionally valid for electrical power grids, where power flows according to physical rules such as Kirchhoff's laws, and load demand. Nevertheless, it serves the purpose of the paper which is to look into the structural properties and apply the NP optimization framework to a complex network of realistic size.

In the energy field, NP by line switching is common practice in problems like line overloads [31], loss and/or cost reductions [61], system security improvements [62], or a combination of them [32]. Examples of line switching in other types of operations are the traffic closure due to road work, accidents or natural disasters, interruption of internet or telecommunication lines. For practical uses, the preliminary analysis propounded can serve for guiding more detailed approaches. Nonetheless, the use of line switching, here taken only as an example of protection measure, must be carefully considered in practice: as seen in our results too, the action of switching off transmission lines during a failure might itself worsen the behavior of the system in some cases.

In this direction, introduction of physical characteristics into abstract modeling approaches is envisaged. Indeed, future research steps will consider the extension of the abstract model of cascading failures "beyond network abstraction" to incorporate physical properties of the engineered network systems which are investigated. This must be done in a way to bridge the modeling close to reality, without over-complicating the model that remains appealing if it requires minimum information and provides prompt results about network vulnerability.

Nevertheless, while cascading model abstracts from the modeling of the realistic power flow, the proposed optimization method can obtain protective strategies of practical feasibility, i.e. suggesting few lines to be turned off to maintain network reliability [34, 55].

\section{CONCLUSIONS}

This paper presents research work investigating the search for optimal network protection strategies against cascading failures within the complex network systems perspective. More specifically, the focus is on the strategy of disconnecting edges in order to avoid or alleviate component overloads that may arise from the occurrence of failures in the infrastructure, which hence may initiate a cascading failure process. Such a 
strategy may be employed either as a manual strategy or as an implemented automatic control strategy, in order to re-route flows so that heavily congested components are relieved.

A new algorithm, namely NSBDE, is proposed for the search of optimal network protection strategies against cascading failures. This algorithm improves the previously proposed MBDE [1], three-fold: 1) in the optimization problem formulation, a third objective function is included to minimize the impact of the switching off operations onto the existing network topology; 2) still in the optimization problem formulation, the final results of cascades, rather than only a short horizon of one step cascading, are used to evaluate the effects of the switch off strategy; 3) in the MOEA, the fast non-dominated sorting mechanisms are incorporated into the MBDE algorithm.

Numerical application to the topological structure $380 \mathrm{kV}$ Italian power transmission network has proved the proposed algorithm to be both promising and practical.

The problem formulation and the optimization algorithm proposed in this work appear to be effective to obtain the optimal network protection strategy for mitigating the cascading failures at a low cost. Further improvements can be carried out in the following directions: 1) extend the applications onto other cascading models with more specific physical properties, e.g. OPA and PSA; 2) comparisons with other evolutionary algorithms, e.g. quantum inspired evolutionary algorithm [63], genetic algorithm [33], simulated annealing [64], etc.

\section{Acknowledgement}

We would like to thank the anonymous reviewers for their valuable comments.

\section{References}

[1] E. Zio, L. R. Golea, and G. Sansavini, "Optimizing protections against cascades in network systems: A modified binary differential evolution algorithm," Reliability Engineering and System Safety, vol.103, pp. 72-83, 2011.

[2] N. Alguacil, M. Carrión, and J. M. Arroyo, "Transmission network expansion planning under deliberate outages," Electrical Power and Energy Systems, vol.31, pp. 553-561, 2009.

[3] J. Johansson and H. Hassel, "An approach for modelling interdependent infrastructures in the context of vulnerability analysis," Reliability Engineering and System Safety, vol.95, no.12, pp. 1335-1344, 2010.

[4] M. L. Sachtjen, B. A. Carreras, and V. E. Lynch, "Disturbances in a power transmission system," Physical Review E, vol.61, pp. 4877-4882, 2000.

[5] J. Glanz and R. Perez-Pena, "90 Seconds That Left Tens of Millions of People in the Dark," in New York Times, ed, 2003.

[6] I. Dobson, B. A. Carreras, and D. E. Newman, "A loading-dependent model of probabilistic cascading failure," Probability in the Engineering and Informational Sciences, vol.19, no.1, pp. 15-32, 2005.

[7] J. Kim and I. Dobson, "Approximating a Loading-Dependent Cascading Failure Model With a Branching Process," IEEE Transactions on Reliability, vol.59, no.4, pp. 691-699, 2010. 
[8] I. Simonsen, L. Buzna, K. Peters, S. Bornholdt, and D. Helbing, "Transient Dynamics Increasing Network Vulnerability to Cascading Failures," Physical Review Letters, vol.100, p. 218701, 2008.

[9] D. J. Watts, "A simple model of global cascades on random networks," in Proceedings of the National Academy of Sciences USA, 2002, vol.99, no.9, pp. 5766-5771.

[10] D. Li, K. Kosmidis, A. Bunde, and S. Havlin, "Dimension of spatially embedded networks," Nature Physics, vol.7, pp. 481- 484, 2011.

[11] Y. Hu, Y. Wang, D. Li, S. Havlin, and Z. Di, "Possible Origin of Efficient Navigation in Small Worlds," Physical Review Letters, vol.106, p. 108701(4), 2011.

[12] D. Li, G. Li, K. Kosmidis, H. E. Stanley, A. Bunde, and S. Havlin, "Percolation of spatially constraint networks," Europhysics Letters, vol.93, no.6, p. 68004(5), 2011.

[13] R. Albert, I. Albert, and G. L. Nakarado, "Structural vulnerability of the North American power grid," Physical Review E, vol.69, 2004.

[14] P. Crucitti, V. Latora, and M. Marchiori, "Model for cascading failures in complex networks," Physical Review E, vol.69, no.4, p. 045104, 2004.

[15] B. A. Carreras, V. E. Lynch, I. Dobson, and D. E. Newman, "Complex Dynamics of Blackouts in Power Transmission Systems," Chaos, vol.14, no.3, pp. 643-652, 2004.

[16] M. Anghel, K. A. Werley, and A. E. Motter, "Stochastic Model for Power Grid Dynamics," presented at the HICSS'07 : Proceedings of the 40th Annual Hawaii International Conference on System Sciences, IEEE Computer Society, Washington, DC, USA, 2007.

[17] X. Yu and C. Singh, "A Practical Approach for Integrated Power System Vulnerability Analysis with Protection Failures," IEEE Transactions on Power Systems, vol.19, no.4, pp. 1811-1820, 2004.

[18] M. Cepin, Assessment of Power System Reliability: Methods and Applications. London, UK: Springer-Verlag, 2011.

[19] A. E. Motter and Y. C. Lai, "Cascade-based attacks on complex networks," Physical Review $E$, vol.66, no.6, 2002.

[20] J. Johansson and H. Hassel, "Comparison of vulnerability and reliability analysis of technical infrastructures," in Proceedings of the European Safety and Reliability Conference - ESREL, Troyes, France, 2011, pp. 2465-2473.

[21] C. M. Rocco and J. E. Ramirez-Marquez, "Vulnerability metrics and analysis for communities in complex networks," Reliability Engineering \& System Safety, vol.96, no.10, pp. 1360-1366, 2011.

[22] C. M. Rocco, J. E. Ramirez-Marquez, and A. D. E. Salazar, "Some metrics for assessing the vulnerability of complex networks: An application to an electric power system," in Proceedings of the European Safety and Reliability Conference - ESREL, Troyes, France, 2011, pp. 2556-2561.

[23] J. Johansson, S. LaRocca, H. Hassel, and S. Guikema, "Comparing Topological Performance Measures and Physical Flow Models for Vulnerability Analysis of Power Systems," in Proceedings of the 11th International Probabilistic Safety Assessment and Management Conference - PSAM 11 \& The Annual European Safety and Reliability Conference-ESREL, Helsinki, Finland, 2012.

[24] S. C. M. Rocco and J. E. Ramirez-Marquez, "Deterministic network interdiction optimization via an evolutionary approach," Reliability Engineering and System Safety, vol.94, pp. 568-576, 2009.

[25] J. M. Arroyo and F. J. Fernández, "A Genetic Algorithm Approach for the Analysis of Electric Grid Interdiction with Line Switching," presented at the Intelligent System Applications to Power Systems, ISAP '09, 15th International Conference on, Curitiba, 2009.

[26] C. M. Rocco, J. E. Ramirez-Marquez, and D. E. Salazar, "Bi and tri-objective optimization in the deterministic networkinterdiction problem," Reliability Engineering and System Safety, vol.95 no.8, pp. 887-896, 2010. 
[27] J. E. Ramirez-Marquez and C. M. Rocco, "Stochastic networkinterdiction optimization via capacitated network reliability modeling and probabilistic solution discovery," Reliability Engineering and System Safety, vol.94, no.5, pp. 913-921, 2009.

[28] J. E. Ramirez-Marquez, C. M. Rocco, and G. Levitin, "Optimal network protection against diverse interdictor strategies," Reliability Engineering and System Safety, vol.96, no.3, pp. 374-382, 2011.

[29] J. E. Ramirez-Marquez and C. M. Rocco, "Vulnerability based robust protection strategy selection in service networks," Computers and Industrial Engineering, vol.63, no.1, pp. 235242, 2012.

[30] C. M. Rocco and J. E. Ramirez-Marquez, "A bi-objective approach for shortest-path network interdiction," Computers \& Industrial Engineering, vol.59, no.2, pp. 232-240, 2010.

[31] G. Granelli, M. Montagna, F. Zanellini, P. Bresesti, R. Vailati, and M. Innorta, "Optimal network reconfiguration for congestion management by deterministic and genetic algorithms," Electrical Power and Energy Systems, vol.76, pp. 549-556, 2006.

[32] W. Shao and V. Vittal, "Corrective switching algorithm for relieving overloads and voltage violations," IEEE Transactions on Power Systems, vol.20, no.4, pp. 1877-1885, 2005.

[33] A. A. Abou EL Ela and S. R. Spea, "Optimal corrective actions for power systems using multi-objective genetic algorithms," Electrical Power and Energy Systems, vol.79, no.5, pp. 722-733, 2009.

[34] E. B. Fisher, R. P. O'Neill, and M. C. Ferris, "Optimal Transmission Switching," IEEE Transactions on Power Systems, vol.23, no.3, pp. 1346-1355, 2008.

[35] M. Li, P. B. Luh, L. D. Michel, Q. Zhao, and X. Luo, "Corrective Line Switching With Security Constraints for the Base and Contingency Cases," IEEE Transactions on Power Systems, vol.27, no.1, pp. 125-133, 2012.

[36] J. E. Ramirez-Marquez, C. M. Rocco, and G. Levitin, "Network Protection Against Diverse Attacks - A Multi-objective Perspective," in Proceedings of 11th International Probabilistic Safety Assessment and Management Conference - PSAM 11 \& The Annual European Safety and Reliability Conference - ESREL, Helsinki, Finland, 2012.

[37] L. Dueñas-Osorio and S. M. Vemuru, "Cascading failures in complex infrastructures systems," Structural safety, vol.31, pp. 157-167, 2009.

[38] K. Deb, A. Pratap, S. Agarwal, and T. Meyarivan, "A fast and elitist multiobjective genetic algorithm: NSGA-II," IEEE Transactions on Evolutionary Computation, vol.6, pp. 182-197, 2002.

[39] L. Wang, X. Fu, M. I. Menhas, and M. Fei, "A Modified Binary Differential Evolution Algorithm," in Life System Modeling and Intelligent Computing, Lecture Notes in Computer Science. vol. 6329, 2010, pp. 49-57.

[40] R. Storn and K. Price, "Differential evolution - A simple and efficient adaptive scheme for global optimization over continuous spaces," International Computer Science Institute, Berkeley, CA.1995.

[41] M. Mahdavi, M. Fesanghary, and E. Damangir, "An improved harmony search algorithm for solving optimization problems," Applied Mathematics and Computation, vol.188, no.2, pp. 1567-1579, 2007.

[42] M. P. Saka, "Optimum design of steel sway frames to BS5950 using harmony search algorithm," Journal of Constructional Steel Research, vol.65, no.1, pp. 36-43, 2009.

[43] Z. W. Geem, J. H. Kim, and G. V. Loganathan, "A new heuristic optimization algorithm: harmony search," Simulation, vol.76, no.2, pp. 60-68, 2001.

[44] A. Ponsich and C. A. Coello, "Differential Evolution performances for the solution of mixed integer constrained Process Engineering problems," Applied Soft Computing, vol.11, no.1, pp. 399-409, 2009.

[45] M. E. J. Newman and M. Girvan, "Finding and evaluating community structure in networks," Physical Review E, vol.69, no.2, 2004. 
[46] E. Zio and G. Sansavini, "Modeling Interdependent Network Systems for Identifying Cascade-Safe Operating Margins," IEEE Transactions on Reliability, vol.60, no.1, pp. 94 $101,2011$.

[47] R. G. Little, "Controlling Cascading Failure: Understanding the Vulnerabilities of Interconnected Infrastructures," Journal of Urban Technology, vol.9, no.1, pp. 109-123, 2002.

[48] L. C. Freeman, S. P. Borgatti, and D. R. White, "Centrality in valued graphs: A measure of betweenness based on network flow," Social Networks, vol.13, no.2, pp. 141-154, 1991.

[49] M. Girvan and M. E. J. Newman, "Community structure in social and biological networks," presented at the Proceedings of National Academy of Sciences of USA, Piscataway, NJ, 2002.

[50] R. W. Floyd, "Algorithm 97: Shortest path," Communications of the ACM, vol.5, no.6, p. 345, 1962.

[51] Y. Sawaragy, H. Nakayama, and T. Tanino, Theory of multiobjective optimization. Orlando, FL: Academic Press, 1985.

[52] V. Rosato, L. Issacharoff, and S. Bologna, "Influence of the topology on the power flux of the Italian high-voltage electrical network," Europhysics Letters 2010 (in press).

[53] E. Zitzler and L. Thiele, "Multiobjective optimization using evolu-tionary algorithms-A comparative case study," in Parallel Problem Solving from Nature (PPSN V), A. E. Eiben, et al., Eds. Berlin, Germany: Springer, 1998, pp. 292-301.

[54] Y. Cao. (2008, Sep. 28). Hypervolume Indicator. Available: http://www.mathworks.com/matlabcentral/fileexchange/19651-hypervolume-indicator

[55] V. M. Bier, E. R. Gratz, N. J. Haphuriwat, W. Magua, and K. R. Wierzbicki, "Methodology for identifying near-optimal interdiction strategies for a power transmission system,"

Reliability Engineering \& System Safety, vol.92, no.9, pp. 1151-1161, 2007.

[56] I. Dobson, B. A. Carreras, V. E. Lynch, and D. E. Newman, "Complex systems analysis of series of blackouts: Cascading failure, critical points, and self-organization," Chaos: An Interdisciplinary Journal of Nonlinear Science, vol.17, no.2, pp. 1-13, 2007.

[57] P. Hines, E. Cotilla-Sanchez, and S. Blumsack, "Do topological models provide good information about electricity infrastructure vulnerability," Chaos, An Interdisciplinary Journal of Nonlinear Science, vol.20, no.3, pp. 1-5, 2010.

[58] J. Salmeron, K. Wood, and R. Baldick, "Analysis of Electric Grid Security Under Terrorist Threat," IEEE Transactions on power systems, vol.19, no.2, pp. 905-912, 2004.

[59] W. Kroger and E. Zio, Vulnerable systems, 1 ed. London: Springer-Verlag, 2011.

[60] E. Zio, L. R. Golea, and C. M. S. Rocco, "Identifying groups of critical edges in a realistic electrical network by multi-objective genetic algorithms," Reliability Engineering \& System Safety, vol.99, pp. 172-177, 2012.

[61] G. Schnyder and H. Glavitsch, "Security enhancement using an optimal switching power flow," IEEE Transactions on Power Systems, vol.5, no.2, pp. 674-681, 1990.

[62] G. Schnyder and H. Glavitsch, "Integrated security control using an optimal power flow and switching concepts," IEEE Transactions on Power Systems, vol.3, no.2, pp. 782-790, 1988.

[63] T. W. Lau, C. Y. Chung, K. P. Wong, T. S. Chung, and S. L. Ho, "Quantum-Inspired Evolutionary Algorithm Approach for Unit Commitment," IEEE Transactions on Power Systems, vol.24, no.3, pp. 1503 - 1512, 2009.

[64] M. Cepin, "Optimization of safety equipment outages improves safety," Reliability Engineering and System Safety, vol.77, no.1, pp. 71-80, 2002. 